\title{
Single Polarization Reflectarray Design Based on an Element with Seven Parallel Dipoles to Generate Independent Beams in three frequency bands.
}

\author{
Rafael Florencio Díaz, Gerardo Pérez Palomino , José Antonio Encinar, Rafael Rodríguez Boix
}

\begin{abstract}
A single layer reflectarray element based on seven parallel dipoles is proposed for single polarization applications. The dipoles of the reflectarray element make it possible a multi-resonant behavior which provides very large phase range, while keeping low loss and low cross-polarization level. A sequential design process of a reflectarray antenna is proposed where the lengths of the dipoles are adjusted in pairs to provide the required phases which generate three independents beams at 20,25 and $30 \mathrm{GHz}$. The simulated radiation patterns of the reflectarray antenna exhibit efficiencies of $80 \%, 62 \%$ and $52 \%$ at 20,25 and $30 \mathrm{GHz}$ respectively. The maximum cross-polar radiation obtained is $29.7 \mathrm{~dB}, 32.9 \mathrm{~dB}$ and $39.1 \mathrm{~dB}$ below the maximum of the copolar beam at 20, 25 and $30 \mathrm{GHz}$ respectively. Bandwidths of $14 \%, 8.2 \%$ and $4.3 \%$ are achieved at center frequencies of 20 , 25 and $30 \mathrm{GHz}$ respectively. These bandwidth results account for a $+/-0.5^{\circ}$ of deviation in the main beam direction, for $2 \mathrm{~dB}$ gain variations in the two first bands, and for a $3 \mathrm{~dB}$ gain variation in the last band.
\end{abstract}

Index Terms-reflectarrays, parallel edge-coupled dipoles, single polarization.

\section{INTRODUCTION}

Printed reflectarrays are planar reflector antennas made of one or more layers of microstrip patch arrays [1]. Printed reflectarrays present several technological advantages when compared with conventional reflectors and phased arrays such as low cost, low weight, improved polarization performance and elimination of complex feed networks [2].

The main drawback of the first prototypes of reflectarray antennas was their narrow bandwidth due to the intrinsic finite bandwidth of the microstrip elements. This drawback has been overcome by the use of multilayered substrates that host either two or three metallization levels of rectangular stacked patches [3], [4], or aperture-coupled patches [5], at the cost of a more complex manufacturing process derived from the bonding of different layers with printed elements. In the last decade, different single polarization broadband reflectarray elements made of multi-resonant elements printed on a single dielectric layer have been proposed for to improve the bandwidth, while keeping a simple manufacturing process at the same time. Some of these single layer multi-resonant elements are parallel dipoles [6]-
[9], and concentric rectangular or cross loops [10]-[12], where the relative lengths of the dipoles or loops are adjusted to improve the phase range and the bandwidth of the reflectarray element. The use of multiple resonances makes it possible to achieve a variation of phase-shift in a range larger than $360^{\circ}$ [13], which can be used as an additional degree of freedom for a further improvement of bandwidth, as demonstrated in [4] and [5].

In this work a single layer reflectarray element based on seven parallel dipoles is proposed for single polarization applications. The reflectarray element made of dipoles shows a multi-resonant behavior which provides very large phase range. This large phase range can't be obtained with the reflectarray elements proposed in [6]-[12]. A sequential design process of a reflectarray antenna is proposed which makes it possible to radiate three independent beams at three different frequencies $(20,25$ and $30 \mathrm{GHz})$. To achieve this performance, the lengths of dipoles are adjusted in pairs to provide the required phases that generate the desired beam direction at each of the three frequencies.

\section{REFLECTARRAY ELEMENT}

The reflectarray element proposed in this work is made of seven parallel dipoles embedded in a double-layered substrate as shown in Fig. 1. The dipoles are introduced to control the phase-shift in one linear polarization. The seven dipoles are printed on the upper side of the bottom dielectric layer. The top layer is placed above the bottom layer to avoid

a

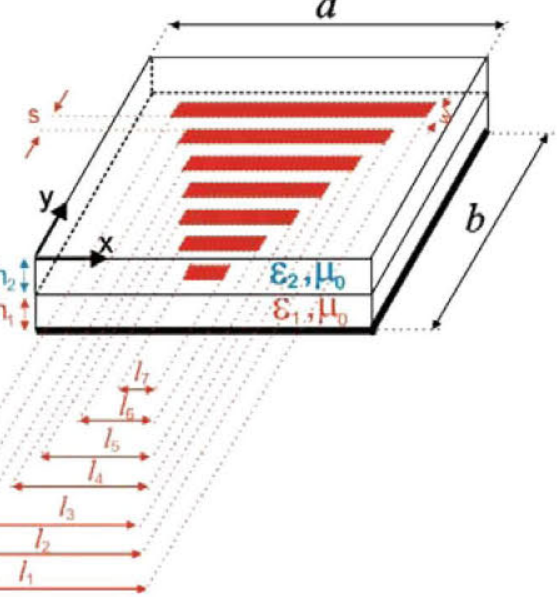

Fig. 1. Reflectarray element made of seven parallel dipoles. 
rust or environmental deterioration of the dipoles. The material used for both the bottom and top layers is the commercial low loss material Rogers 4003C. Honeycomb can also be used instead for the bottom layer to reduce the mass in space antennas, and in this case the dipoles would be printed on the lower side of the top layer. If the complex permittivity of the $i$-th layer of the double-layered substrate is defined as $\varepsilon_{i}=\varepsilon_{0} \varepsilon_{r i}\left(1-j \cdot \tan \delta_{i}\right)-i=1,2$ - (see Fig. 1), then $\varepsilon_{r l}=$ $\varepsilon_{r 2}=3.55, \tan \delta_{1}=\tan \delta_{2}=0.0027$. Also, the thicknesses of the layers are $h_{1}=0.5 \mathrm{~mm}$ and $h_{2}=1.5 \mathrm{~mm}$. The dimensions of the unit cell are $a=5 \mathrm{~mm}$ and $b=5.77 \mathrm{~mm}$ (which correspond to $0.33 \lambda$ and $0.38 \lambda$ at $20 \mathrm{GHz}, 0.42 \lambda$ and $0.48 \lambda$ at $25 \mathrm{GHz}$, and $0.5 \lambda$ and $0.58 \lambda$ at $30 \mathrm{GHz}$ ). These values of $a$ and $b$ have been chosen to provide both enough room for the allocation of the dipoles and enough range of phase-shift at the design frequencies $(20,25$ and $30 \mathrm{GHz})$ and, at the same time, to avoid the appearance of grating lobes. The rest of the geometrical parameters have been adjusted to provide a smooth and linear behavior of the phase curves for variations of the lengths of the dipoles, the final dimensions being $l_{1}=$ $l, l_{2}=0.85 l, l_{3}=0.77 l, l_{4}=0.68 l, l_{5}=0.57 l, l_{6}=l_{7}=0.5 l, w=$ $0.5 \mathrm{~mm}, s=0.3 \mathrm{~mm}$.

A home-made electromagnetic code that applies the Method of Moments in the Spectral Domain (MoM-SD) has been used for the analysis of the reflectarray cell in a periodic environment. Basis functions accounting for edge singularities have been used in the approximation of the current density of the dipoles [14]. The reflectarray cell is characterized by means of the reflection matrix $\mathbf{R}$, which contains the co-polar and cross-polar reflection coefficients for the two linear polarizations parallel to the axes $\mathrm{x}$ and $\mathrm{y}$ shown in Fig. 1. The $\mathbf{R}$ matrix relates the cartesian complex components of the reflected and incident tangential electric field on the reflectarray cell in a periodic environment as shown below

$$
\left(\begin{array}{l}
E_{x}^{r e f} \\
E_{y}^{r e f}
\end{array}\right)=\left(\begin{array}{ll}
R_{x x} & R_{x y} \\
R_{y x} & R_{y y}
\end{array}\right) \cdot\left(\begin{array}{l}
E_{x}^{i n c} \\
E_{y}^{i n c}
\end{array}\right)
$$

Fig. 2 shows the results for the magnitude and phase of $\mathrm{R}_{\mathrm{xx}}$ under oblique incidence $\theta_{i}=19^{\circ}, \phi_{i}=0^{\circ}, \theta_{i}$ and $\phi_{i}$ being the spherical angular coordinates of the incidence direction.
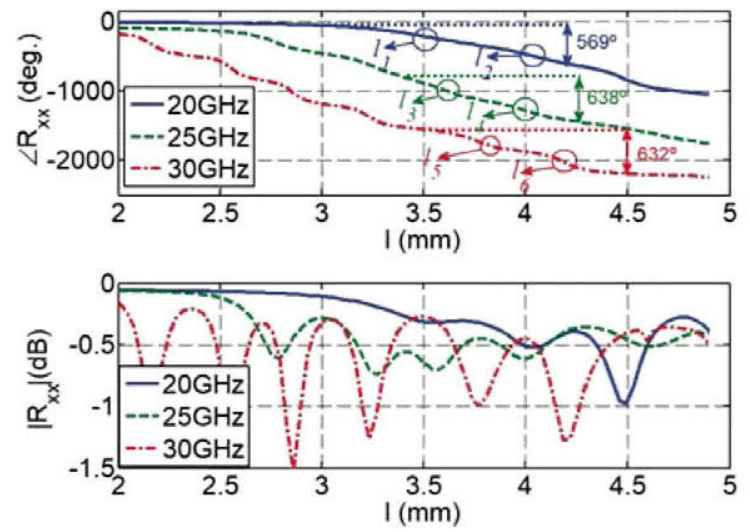

Fig. 2. Magnitude and phase of the cell reflection coefficient $R_{x x}$ with respect to the length of dipoles at different frequencies under oblique incidence $\left(\theta_{i}=19^{\circ} \phi_{i}=0^{\circ}\right)$.
The results of Fig. 2 show that the element of Fig. 1 presents low losses (typically below $1 \mathrm{~dB}$ ), and a linear and smooth behavior of the phase curves with very large phase range. In fact, a phase range of $569^{\circ}$ is obtained due to the resonances of the the dipoles with lengths $l_{1}$ and $l_{2}$ at $20 \mathrm{GHz}$, a phase range of $638^{\circ}$ is obtained due to the dipoles with lengths $l_{3}$ and $l_{4}$ at $25 \mathrm{GHz}$, and finally, a phase range of $632^{\circ}$ is obtained due to the dipoles with lengths $l_{5}, l_{6}$ and $l_{7}$ at 30 GHz. This means that a design of a reflectarray antenna with different radiation patterns at the three frequencies can be achieved by means of a sequential adjustment of the lengths of the dipoles in three steps. In the first step of the design, the lengths $l_{1}$ and $l_{2}$ of the dipoles are adjusted in each reflectarray element to provide the required phase-shift at $20 \mathrm{GHz}$. This adjustment is carried out by sweeping the parameter $l$ while keeping the relations $l_{1}=l$ and $l_{2}=0.85 l$. In this first step the rest of the dipoles with lengths $l_{3}, l_{4}, l_{5}, l_{6}$ and $l_{7}$ of each reflectarray element are removed. In the second step of the design, the lengths of dipoles $l_{3}$ and $l_{4}$ are adjusted in each reflectarray element to provide the required phase-shift at $25 \mathrm{GHz}$. This adjustment is carried out by sweeping the parameter $l$ while keeping the relations $l_{3}=$ $0.77 l$ and $l_{4}=0.68 l$. In this second step the lengths $l_{1}$ and $l_{2}$ previously adjusted for each reflectarray element during the first step are kept constant. Also, in this second step the dipoles with lengths $l_{5}, l_{6}$ and $l_{7}$ of each reflectarray element are removed. Finally, in the third step the lengths of the dipoles $l_{5}, l_{6}$ and $l_{7}$ are adjusted in each reflectarray element to provide the required phase-shift at $30 \mathrm{GHz}$. This adjustment is carried out by sweeping the parameter $l$ while keeping the relations $l_{5}=0.57 l, l_{6}=l_{7}=0.5 \mathrm{l}$. In this third step the lengths $l_{1}, l_{2}, l_{3}$ and $l_{4}$ previously adjusted for each reflectarray element during the first two steps are kept constant

Fig. 3 shows the results obtained for the magnitude of the cross-polar coefficient $\mathrm{R}_{\mathrm{yx}}$ under oblique incidence $\theta_{i}=$ $25^{\circ}, \phi_{i}=25^{\circ}$ ). The results show that $R_{y x}$ goes to zero for two

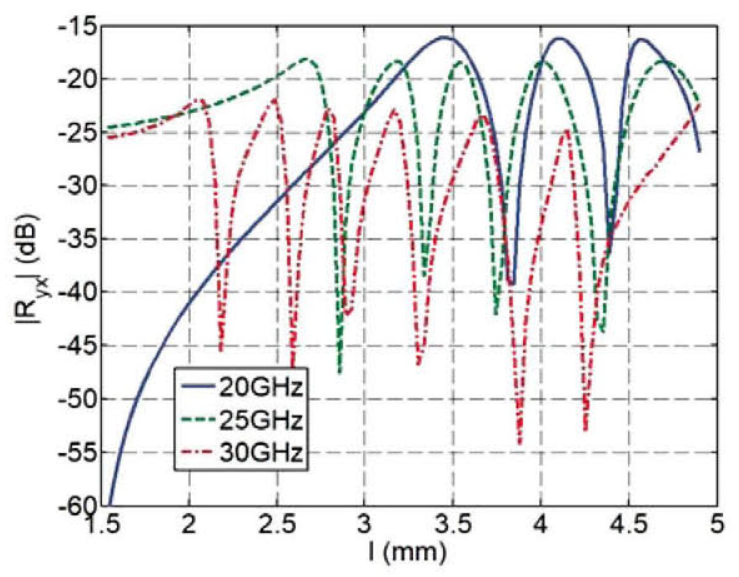

Fig. 3. Magnitude of the cross-polar cell reflection coefficient $R_{y x}$ with respect to the length of dipoles at different frequencies under oblique incidence $\theta_{i}=25^{\circ} \phi i=25^{\circ}$.

different values of the dipole lengths at $20 \mathrm{GHz}$. The number 
of zero-crossings increases to 4 at $25 \mathrm{GHz}$, and to 6 at 30 GHz. Note that Fig. 2 also shows there are 2 resonance lengths at $20 \mathrm{GHz}, 4$ resonance lengths at $25 \mathrm{GHz}$ and 6 resonance lengths at $30 \mathrm{GHz}$. So, the number of zerocrossings in Fig. 3 is related with the number of resonance lengths appearing in the phase curves shown in Fig. 2. According to [15], this high number of zero-crossings for $\mathrm{R}_{\mathrm{yx}}$

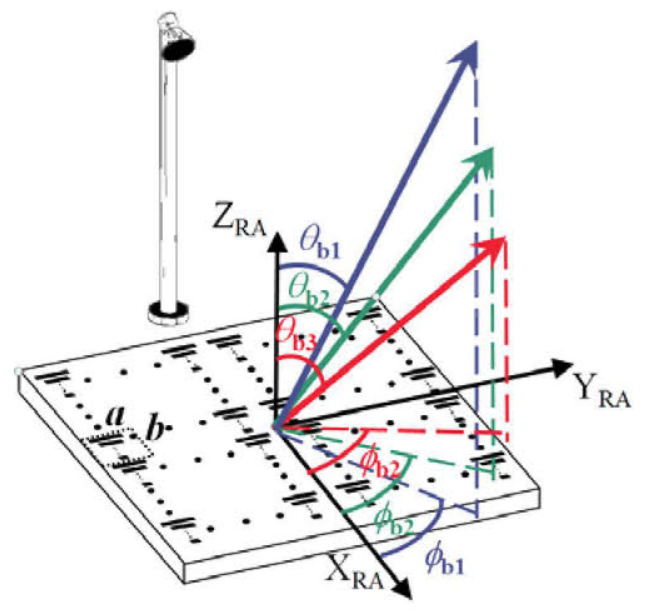

Fig. 4. Coordinate system with origin at the reflectarray center and beam directions.

ensures a low level of cross-polar radiation in a reflectarray antenna based on this type of element.

\section{REFLECTARRAY DESIGN}

A reflectarray antenna made of elements such as that shown in Fig. 2 has been designed to produce a focused beam in the directions $\theta_{b 1}=19^{\circ}, \varphi_{b 1}=0^{\circ}$ at $20 \mathrm{GHz}, \theta_{b 2}=25^{\circ}$, $\varphi_{b 2}=0^{\circ}$ at $25 \mathrm{GHz}$ and $\theta_{b 3}=32^{\circ}, \varphi_{b 3}=0^{\circ}$ at $30 \mathrm{GHz}$ for Xpolarization (see Fig. 4 for the definition of the angles $\theta_{b i}$ and $\left.\varphi_{b i}-i=1,2,3-\right)$. The reflectarray element has been characterized as described in the previous section. The reflectarray is rectangular and consists of 525 elements arranged in a $25 \times 21$ grid with cell size $5 \mathrm{~mm} \times 5.77 \mathrm{~mm}$. The reflectarray is illuminated by a feed-horn with its phase center located at coordinates $x_{R A}=-63.9 \mathrm{~mm}, y_{R A}=0 \mathrm{~mm}$ and $z_{R A}=185.5 \mathrm{~mm}$ with respect to a coordinate system with origin at the center of the reflectarray (see the coordinate system in Fig. 4). The direction of the radiated beam at 20 $\mathrm{GHz}-\theta_{b l}=19^{\circ}, \varphi_{b l}=0^{\circ}$ - has been chosen to be the specular reflection direction of the beam arriving at the reflectarray center from the feed horn, which prevents the beam squint effect from appearing at frequencies around $20 \mathrm{GHz}$ [16]. Since the radiated beam directions at 25 and $30 \mathrm{GHz}$ are not the specular reflection directions of the feeding beam, beam squint effects are to be expected around those frequencies.

The required phase distribution to produce a collimated beam in the direction $\theta_{b 1}=19^{\circ}, \varphi_{b 1}=0^{\circ}$ at $20 \mathrm{GHz}, \theta_{b 2}=25^{\circ}$, $\varphi_{b 2}=0^{\circ}$ at $25 \mathrm{GHz}$ and $\theta_{b 3}=32^{\circ}, \varphi_{b 3}=0^{\circ}$ at $30 \mathrm{GHz}$ for Xpolarization are shown in Fig. 5(a), Fig. 5(b) and Fig. 5(c) respectively. The radiation pattern of the feed is modeled as a function $\cos ^{20}(\theta)$, which provides an illumination level at the reflectarray edges $10 \mathrm{~dB}$ below the maximum.

The reflectarray design follows the three steps described in section II. In the first step the parameter $l$ involved in $l_{1}$ and $l_{2}$ is adjusted to match the required phase-shift shown in Fig. 5(a) to the phase of the reflection coefficient $R_{x x}$ in each

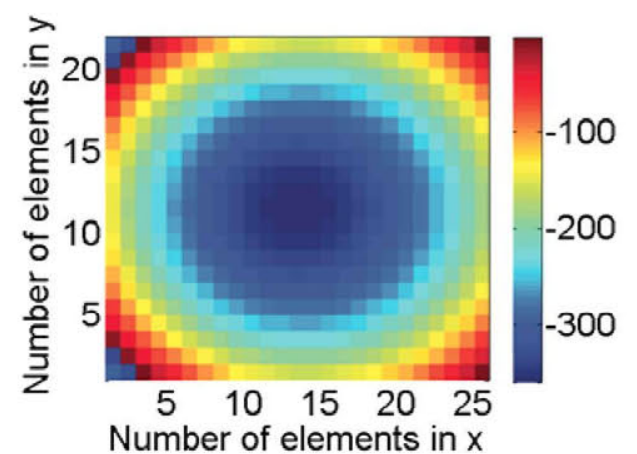

(a)

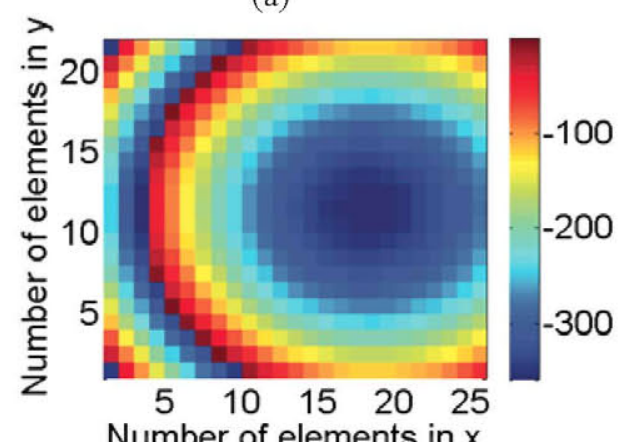

(b)

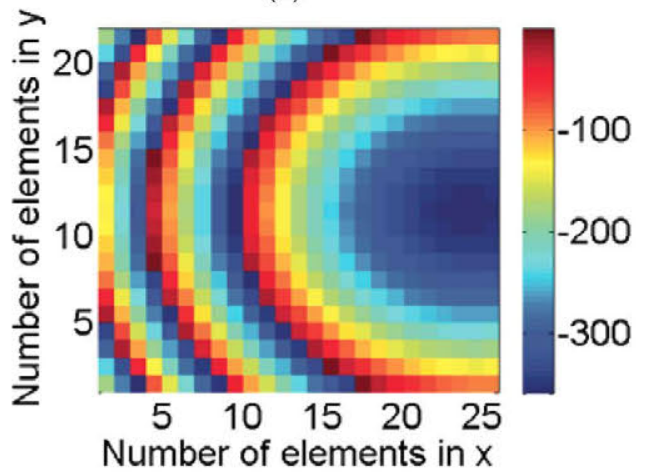

Fig. 5. Required phase shift to produce a collimated beam for Xpolarization in the direction (a) $\theta_{b 1}=19^{\circ}$ and $\phi_{b l}=0^{\circ}$ at $20 \mathrm{GHz}$, (b) $\theta_{b 2}=25^{\circ}$ and $\phi_{b 2}=0^{\circ}$ at $25 \mathrm{GHz}$, and (c) $\theta_{b 3}=32^{\circ}$ and $\phi_{b 3}=0^{\circ}$ at 30 GHz.

reflectarray element at $20 \mathrm{GHz}$. In the second stage, the parameter $l$ involved in $l_{3}$ and $l_{4}$ is adjusted to match the required phase-shift shown in Fig. 5(b) to the phase of the reflection coefficient $R_{x x}$ in each reflectarray element at $25 \mathrm{GHz}$. Finally, in the third stage, the parameter $l$ involved in $l_{5}, l_{6}$ and $l_{7}$ is adjusted to match the required phase-shift shown in Fig. 5(c) to the phase of the reflection coefficient $\mathrm{R}_{\mathrm{xx}}$ in each reflectarray element at $30 \mathrm{GHz}$. In all the three 
steps of the design process, local periodicity is assumed and the angle of incidence in each element of the reflectarray is explicitly considered.

\section{NUMERICAL RESULTS}

Figs. 6(a) and (b) show the elevation and azimuth cuts of the computed radiation patterns for the reflectarray designed in Section III at 20,25 and $30 \mathrm{GHz}$. These radiation patterns have been computed in terms of the electric and magnetic equivalent currents existing on the reflectarray surface, which are provided by MoM-SD for each reflectarray element (technique II in [17]). The antenna gain is computed in terms of the power radiated by the feed, and the radiation patterns are computed with reference to the isotropic antenna (in $\mathrm{dBi}$ ) [1]. The numerical results obtained for the radiation patterns indicate a gain of $27.13 \mathrm{dBi}$ (i.e., an efficiency of $80 \%$ ) at $20 \mathrm{GHz}, 27.92 \mathrm{dBi}$ (i.e., an efficiency of $62 \%$ ) at 25 $\mathrm{GHz}$, and $28.72 \mathrm{dBi}$ (i.e, an efficiency of $52 \%$ ) at $30 \mathrm{GHz}$. Moreover, the radiation patterns exhibit very low cross polarization. In fact, the maximum levels of cross-polar radiation have been found to be $29.7 \mathrm{~dB}, 32.9 \mathrm{~dB}$ and 39.1 $\mathrm{dB}$ below the co-polar maxima at 20,25 and $30 \mathrm{GHz}$ respectively. The cross-polarization in the elevation plane in Fig. 6(a) is lower than $-20 \mathrm{dBi}$ because the elevation plane is a symmetry plane. Note that the cross-polar radiation level is lower as the frequency increases. This result is to be expected since Fig. 3 shows an increment of the number of zero-crossings with increasing frequency, and the larger the number of zero-crossings, the lower the cross-polar radiation [15].

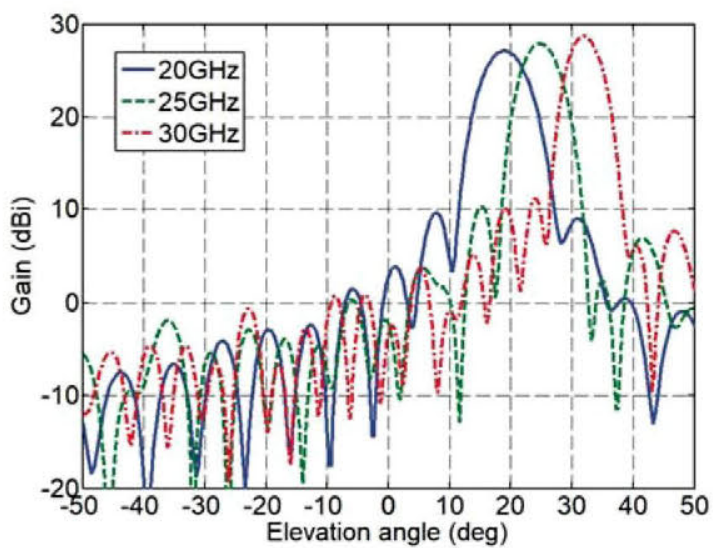

(a)

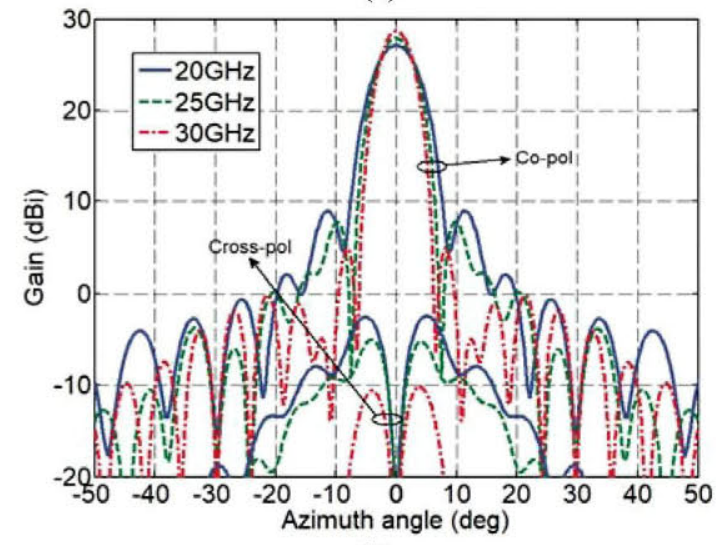

(b)

Fig. 6. Main cuts of the radiation patterns generated at 20,25 and 30 $\mathrm{GHz}$ by the reflectarray designed for X-polarization. (a) Elevation cut. (b) Azimuth cut.
Fig. 7(a) shows both the gain and the maximum level of cross-polar radiation as a function of frequency for $\mathrm{X}$ polarization. Fig. 7(b) shows the spherical angular coordinate $\theta_{b, \max }$ giving the main beam direction as a function of frequency (see Fig. 4). The results shown in Figs. 7(a) and (b) show a variation of $+/-0.5^{\circ}$ in the main beam direction in the range $19-21.8 \mathrm{GHz}$ with $2 \mathrm{~dB}$-gain varitation (14\% bandwidth), in the range 24.9-27 $\mathrm{GHz}$ with 2dB-gain variation (8.4\% bandwidth), and in the range $29.5-30.8 \mathrm{GHz}$ with $3 \mathrm{~dB}$-gain variation $(4.3 \%$ bandwidth). Note that the main limitation of the bandwidth is due to the variation of $+/$ $0.5^{\circ}$ in the main beam direction. This variation of $+/-0.5^{\circ}$ causes a smaller bandwidth around the design frequencies of 25 and $30 \mathrm{GHz}$, and the reason for this is that the main beam direction is not the specular reflection direction of the feeding beam at those frequencies, which is the origin of beam squint [16]. A wider bandwidth is to be expected at 20 $\mathrm{GHz}$ where the main beam direction is the specular reflection direction of the feeding beam.

\section{CONCLUSION}

A reflectarray antenna made of reflectarray elements based on seven parallel dipoles has been designed to

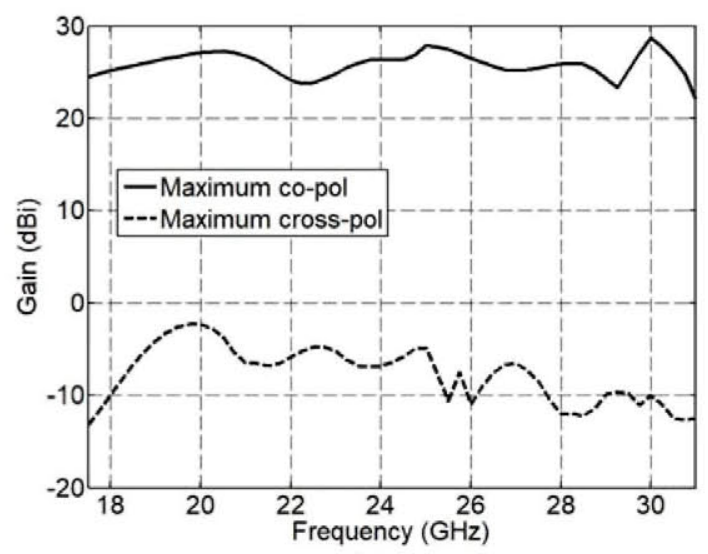

(a)

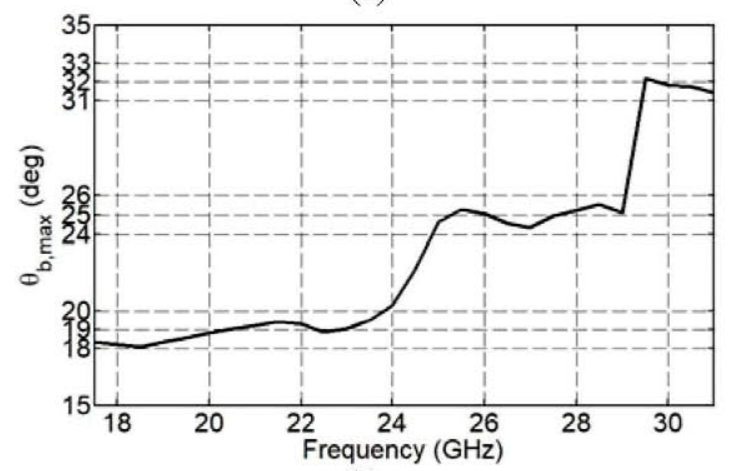

(b)

Fig. 7. (a) Maxmimum gain of the copolar and cross-polar radiation as a function of frequency. (b) Main beam direction as a function of frequency. 
generate three independent beams for single polarization at 20,25 and $30 \mathrm{GHz}$ respectively. A sequential design process is proposed where the lengths of dipoles are adjusted in pairs to provide the required phases that generate the three independents beams. The simulated radiation patterns of the designed reflectarray antenna exhibit efficiencies of $80 \%$, $62 \%$ and $52 \%$, and cross-polar maxima below the main beam of $29.7 \mathrm{~dB}, 32.9 \mathrm{~dB}$ and $39.1 \mathrm{~dB}$, at 20,25 and $30 \mathrm{GHz}$ respectively. A detailed study has been carried out on the variation of both the gain and the main beam direction as a function of frequeny. The results show variations of $+/-0.5^{\circ}$ in the main beam direction in the range $19-21.8 \mathrm{GHz}$ with $2 \mathrm{~dB}$-gain varitation ( $14 \%$ bandwidth), in the range of 24.9 $27.0 \mathrm{GHz}$ with $2 \mathrm{~dB}$-gain variation ( $8.4 \%$ bandwidth), and in the range $29.5-30.8 \mathrm{GHz}$ with $3 \mathrm{~dB}$-gain variation $(4.3 \%$ bandwidth). The reduction of bandwidth with increasing frequency is attributed to beam squint caused by the fact that the main beam direction is not the specular reflection direction of the feeding beam.

\section{ACKNOWLEDGMENT}

This work has been supported by European Space Agency under ESTEC Contract No. 4000117113/16/NL/AF, by the Spanish Ministry of Economy and Competitiveness (project CYCIT TEC2016-75103-C2-1-R), and by 'Junta de Andalucía' (project P12-TIC-1435).

\section{REFERENCES}

[1] J. Huang and J. A. Encinar, Reflectarray antennas. Piscataway, NJ/New York: IEEE Press/Wiley, 2008.

[2] D D.M. Pozar, S.D. Targonski, and H.D. Syrigos, "Design of millimeter wave microstrip reflectarray," IEEE Trans. Antennas Propagat. vol. 45, No. 2, pp. 287-296, February 1997.

[3] J. A. Encinar, "Design of two-layer printed reflectarrays using patches of variable size," IEEE Trans. Antennas Propagat. vol. 49 , pp. 1403--1410, Oct. 2001.

[4] J. A. Encinar, J. A. Zornoza, "Broadband design of three--layer printed reflectarrays," IEEE Trans. on Antennas and Propag. Vol. 51, No. 7, July 2003, pp. 1662-1664.

[5] E. Carrasco, J. A. Encinar and M. Barba, "Bandwidth improvement in large reflectarrays by using true-time-delay," IEEE Trans. Antennas Propagat. Vol. 56, pp. 2496-2503, Aug. 2008.

[6] S. Dieter, C. Fischer, W. Menzel, "Single-layer unit cells with optimized phase angle behaviour," Proceedings of the 3rd European Conference on Antennas and Propagation, EuCAP 2009, pp.11491153, March 2009.

[7] R. Florencio, R. R. Boix, J. A. Encinar, E. Carrasco, M. Arrebola and V. Losada, "Comparative study of reflectarrays based on cells with three coplanar parallel and reflectarrays based on cells with three stacked patches," Proc. 6th Edition EUCAP, Prague, Czech Republic, Mar. 2012.

[8] R. Florencio, R. R. Boix, E. Carrasco, J. A. Encinar, M. Barba and G. Pérez-Palomino, "Broadband Reflectarrays Made of Cells with Three Coplanar parallel dipoles," Microwave Opt. Technol. Lett., vol. 56, No. 3, pp. 748-753, March 2014.

[9] J. H. Yoon, Y. J. Yoon, W. Lee, and J. So, "Broadband microstrip reflectarray with five parallel dipole elements," IEEE Antennas Wireless Propag. Lett., vol. 14, pp. 1109-1112, 2015.

[10] M.R. Chaharmir, J. Shaker, M. Cuhaci, and A. Ittipiboon, "A broadband reflectarray antenna with double square rings," Microw. Opt. Technol. Lett., 2006, 48, (7), pp. 1317-1320.
[11] M. R. Chaharmir and J. Shaker, "Broadband reflectarray with combination of cross and rectangle loop elements," Electron. Lett., vol. 44, No. 11, pp. 658-659, 2008.

[12] M. R. Chaharmir, J. Shaker, and H. Legay, "Broadband design of a single layer reflectarray using multi cross loop elements", IEEE Trans. Antennas Propagat. Vol. 57, pp. 3363-3366, Oct. 2008.

[13] K, H., Sayidmarie and M. E. Bialkowski, "Fractal unit cells of increased phasing range and low slopes for single-layer microstrip reflectarrays," IET Microw. Antennas Propag., Vol. 5, pp. 1371-1379, Dec. 2011.

[14] R. Florencio, R. R. Boix, E. Carrasco, J. A. Encinar, and V. Losada, "Efficient numerical tool for the analysis and design of reflectarrays based on cells with three parallel dipoles", Microw. Opt. Technol. Lett., vol. 55, no. 6, pp. 1212-1216, June 2013.

[15] C. Tienda, J. A. Encinar, M. Barba, and M. Arrebola, "Reduction of cross-polarization in offset reflectarrays using two layers of varyingsized patches", Microw. Opt. Technol. Lett., Vol. 54, No. 11, pp. 2449-2454, Nov. 2012.

[16] S.D.Targonski, D.M. Pozar, "Minimization of beam squint in microstrip reflectarrays using an offset feed," Antennas and Propagation Society International Symposium, 1996. AP-S. Digest, vol.2, no., pp.1326-1329 vol.2, 21-26 July 1996.

[17] M. Zhou, S. B. Sorensen, O. S. Kim, E. Jorgensen, P. Meincke, O. S Kim and $O$. Breinbjerg, "An accurate technique for calculation of radiation from printed reflectarrays," IEEE Trans. Antennas Propagat., vol. 10, pp. 1081-1084, Oct. 2011. 